\section{Recruitment of the SWI/SNF chromatin remodeling complex by transcriptional activators}

\author{
Natalya Yudkovsky, ${ }^{1,4}$ Colin Logie, ${ }^{2,4}$ \\ Steven Hahn, ${ }^{3}$ and Craig L. Peterson ${ }^{2,5}$ \\ ${ }^{1}$ Molecular and Cellular Biology Program, University \\ of Washington, The Fred Hutchinson Cancer Research \\ Center ${ }^{3}$ Fred Hutchinson Cancer Research Center \\ and the Howard Hughes Medical Institute, Seattle \\ Washington 98109 USA; ${ }^{2}$ Program in Molecular Medicine \\ and Department of Biochemistry and Molecular Biology, \\ University of Massachusetts Medical School, \\ Worcester, Massachusetts 01605 USA
}

SWI/SNF is a chromatin remodeling complex that facilitates expression of a number of yeast genes. Here we demonstrate that SWI/SNF can be recruited from yeast nuclear extracts by a transcriptional activator. Recruitment is dependent on an activation domain but not on promoter sequences, TBP, or RNA polymerase II holoenzyme. We also show that acidic activation domains can target SWI/SNF remodeling activity. These results demonstrate that SWI/SNF activity can be targeted by gene-specific activators and that this recruitment can occur independently of Pol II holoenzyme.

Received June 29, 1999; revised version accepted August 6, 1999.

The regulation of transcription initiation requires that transcription factors function in the context of eukaryotic chromatin. Two classes of chromatin remodeling enzymes have been identified that facilitate transcription from chromatin templates in vivo, the histone acetyltransferases and the ATP-dependent remodeling enzymes. The founding member of the transcription-associated histone acetyltransferases is yeast Gcn5p, which was initially identified as a transcriptional coactivator (Georgakopoulos and Thireos 1992; Marcus et al. 1994) and then subsequently as the catalytic subunit of several histone acetyltransferase complexes (Grant et al. 1997; Pollard and Peterson 1997; Saleh et al. 1997). The yeast SWI/SNF complex is a paradigm of the ATP-dependent chromatin remodeling enzymes and is required for expression of a number of genes and the full functioning of a variety of transcriptional activators (Burns and Peterson 1997). Genetic studies initially suggested that the transcriptional requirement for SWI/SNF reflected the ability of the complex to antagonize chromatin-medi-

[Key Words: SWI-SNF; chromatin; transcription; gene regulation; holoenzyme]

${ }^{4}$ These two authors contributed equally to this work.

${ }^{5}$ Corresponding author.

E-MAIL craig.peterson@umassmed.edu; FAX (508) 856-4289. ated transcriptional repression (Winston and Carlson 1992; Kruger et al. 1995). Purified yeast SWI/SNF is a 2-MD enzyme that can use the energy of ATP hydrolysis to increase the accessibility of nucleosomal DNA to DNase I (Côté et al. 1994), restriction enzymes (Logie and Peterson 1997, 1999), or DNA-binding transcription factors in vitro (Côté et al. 1994; Utley et al. 1997).

The inactivation of SWI/SNF results in a decrease in transcription of only a small subset of yeast genes (Burns and Peterson 1997; Holstege et al. 1998), indicating that SWI/SNF might need to be targeted to particular genes and to specific nucleosomes in vivo. Three different models have been proposed to explain SWI/SNF. The nontargeting model proposes that SWI/SNF introduces transient changes in chromatin structure by a catalytic and random fashion throughout the genome, and that persistent, targeted changes in chromatin only occur in the presence of a DNA-binding transcription factor (Owen-Hughes et al. 1996). Alternatively, SWI/SNF might be targeted to specific genes by virtue of its association with an RNA polymerase II (Pol II) holoenzyme that was itself recruited to the promoter region via transcriptional activator proteins. Indeed, both yeast SWI/ SNF and its mammalian homolog, BRG1, have been reported to copurify with Pol II holoenzyme (Wilson et al. 1996; Cho et al. 1998; Neish et al. 1998). However, other Pol II holoenzyme preparations do not contain SWI/SNF, suggesting that only a fraction of holoenzyme contains SWI/SNF (Myers et al. 1998). Finally, SWI/SNF might be targeted through direct interactions with gene-specific activators. Consistent with this model, yeast SWI/SNF can interact with the mammalian glucocorticoid receptor in yeast whole-cell extracts (Yoshinaga et al. 1992), and recruitment of SWI/SNF to the HO promoter in vivo requires the SWI5 activator (Cosma et al. 1999).

Here we have tested directly whether transcriptional activators can recruit the SWI/SNF complex and if recruitment requires an obligatory association with a Pol II holoenzyme. We find that a DNA-bound activator can recruit SWI/SNF from a yeast nuclear extract (NE) under conditions in which RNA Pol II holoenzyme and other general transcription factors (GTFs) are not sequestered. Moreover, the acidic activators, GAL4-AH and GAL4VP16, can target SWI/SNF remodeling activity in a purified system containing only DNA, histones, SWI/SNF, and activator proteins. By contrast, the GAL4 DNAbinding domain or a nonacidic activator, GAL4-proline, are unable to recruit SWI/SNF activity. These results indicate that SWI/SNF may be targeted to a subset of yeast genes by direct interactions with gene-specific transcriptional activators.

\section{Results and Discussion}

Recruitment of the SWI/SNF complex can occur independently of RNA Pol II holoenzyme and TBP

To investigate the targeting of SWI/SNF to a promoter, 
we used yeast nuclear transcription extracts (NEs) in combination with modified His 4 promoter templates immobilized on magnetic beads (Fig. 1A; Ranish et al. 1999). Preinitiation complexes (PICs) were formed by incubating NE with the immobilized template, followed by washing of the complexes, and liberation of the PIC with PstI restriction enzyme digestion. In this system, PIC assembly depends on the presence of a promoter, TATA box, TBP, TFIIA, and holoenzyme subunits, and is modestly stimulated by activators (Ranish et al. 1999).

Figure 1B shows a Western blot of a PIC assembly experiment with Gal4-AH. Two subunits of the SWI/SNF complex, Swi3p and Snf5p, were recruited to the template DNA with extracts from wild-type cells (lane 1). To determine whether SWI/SNF is recruited to DNA as part of Pol II holoenzyme, we used a NE made from a srb2 deletion (srb2 2$)$ strain in our immobilized template assay. This extract is defective for in vitro transcription in this assay, and its activity can be partially restored by the addition of recombinant Srb2 (rSrb2) (Ranish et al. 1999). In addition, deletion or mutation of genes that encode holoenzyme components, such as Srb2p, prevents the recruitment of the entire holoenzyme complex to the promoter, but allows recruitment of TFIID and TFIIA in vitro (Ranish et al. 1999). Figure 1B shows the levels of Rpb3p, a Pol II subunit, and Gall1p, a holoenzyme subunit, are severely decreased in the case of the $\operatorname{srb} 2 \Delta \mathrm{NE}$, as compared with wild type (lane 1 vs. lane 2). Importantly, the levels of both of these components increased significantly on the addition of rSrb2 (lane 3). Although the levels of Swi3p and Snf5p were lower in the case of the srb2s extract, as compared with wild type, their binding was not stimulated by the addition of rSrb2 (Fig. 1B, lanes 1-3). Thus, the lower binding of SWI/SNF in this extract is probably due to a lower specific activity of this extract and not a consequence of the srb2 mutation. To test whether SWI/SNF was recruited to the template by a GTF not in the holoenzyme, such as TFIID, we used

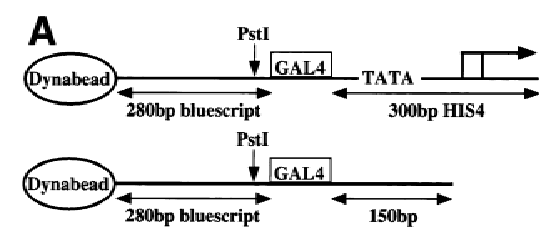

Figure 1. SWI/SNF is recruited to promoters in the immobilized template assay independently of holoenzyme and TBP. (A) Immobilized templates used in this study. (Top) The wild-type template contains the HIS4 core promoter and transcription start sites; (bottom)

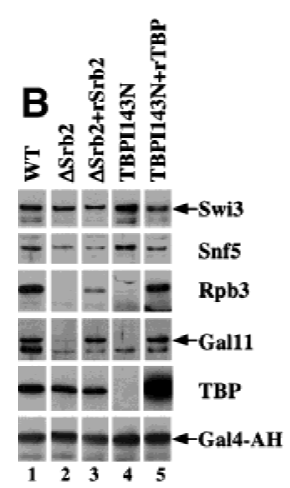
the core promoter, including the TATA box, was deleted and replaced by downstream nonpromoter sequences to create the $\Delta$ Promoter template. $(B)$ Recruitment of Swi3p and Snf5p in wild-type and mutant NEs. PIC assembly was performed with the nuclear extracts indicated at top and analyzed by Western blotting using antibodies against the components indicated at right. All reactions included the activator Gal4-AH. rSrb2 (200 $\mathrm{ng}$ ) and rTBP (400 ng) were added where indicated. an extract made from a TBP temperature-sensitive mutant strain in the immobilized template assay. The I143N mutation in TBP abolishes TBP-DNA binding and disrupts all PIC formation (Fig. 1B, lane 4; Reddy and Hahn 1991). NE made from this strain is defective for in vitro transcription assays, but its activity can be restored by the addition of rTBP (Ranish et al. 1999). This TBP mutation did not affect the recruitment of either Swi3p or Snf5p to the promoter, and addition of rTBP did not stimulate the levels of these factors as it did Rpb3p and Gal11p (Fig. 1B, lanes 4,5). We therefore conclude that in this transcription system, the recruitment of SWI/SNF to the promoter occurs independently of the recruitment of holoenzyme and of other GTFs.

\section{SWI/SNF is recruited to DNA by activators}

Next we tested directly whether SWI/SNF was recruited to the templates by activator in the yeast NEs. Assays were performed either without activator, with the Gal4(1-94) DNA-binding domain, or with Gal4-AH or Gal4-VP16, with both wild-type and TBPI143N NEs. In the wild-type extract, Gal4-AH stimulated recruitment of Rpb3p, Srb4p, TFIIB, and Toa2p 4- to 10-fold, as compared with recruitment in the absence of activator (Fig. 2, lane 2 vs. lane 6). Gal4-AH did not significantly recruit TBP (Ranish et al. 1999). Gal4-VP16, however, stimulated recruitment of all these components 2- to 20-fold (Fig. 2, lane 2 vs. lane 8). Recruitment of Swi3p and Snf5p by these activators was stronger than the recruitment of holoenzyme components. Gal4-AH increased Swi3p and Snf5p levels >13-fold, whereas Gal4VP16 increased Swi3p and Snf5p levels $>21$-fold; both Swi3p and Snf5p were barely detectable in the absence of activator (Fig. 2, lanes 2,6,8). In contrast, the binding of subunits of two other SWI/SNF-like complexes, Iswlp, a component of ISW1 (Tsukiyama et al. 1999), and Sth1p, a component of RSC (Cairns et al. 1996), was constitutive and was not influenced by the presence or absence of activators, promoter sequences, or Pol II holoenzyme (Fig. 2; data not shown). The decrease in Sth1p levels seen on the $\Delta$ Promoter template is most likely due to the fact that this template is $\sim 150$ bp shorter than the wildtype template. To show that the recruitment of SWI/ SNF was dependent on the activation domains, we used Gal4(1-94) as a control (Fig. 2, lane 4). Although it weakly stimulated binding of Rpb3p, Srb4p, TFIIB, and Toa2p levels, we saw no stimulation of either Swi3p or Snf5p recruitment. Both Gal4-AH and Gal4-VP16 stimulated Swi3p and Snf5p recruitment to the same extent in TBPI143N NE as in wild type (Fig. 2, lanes $10,12,14)$. Importantly, Swi3p and Snf5p recruitment was unaffected by the addition of rTBP to restore PIC assembly (Fig. 2, lanes 16,18,20). In the case of Gal4-AH, SWI/SNF recruitment clearly occurred in the absence of recruitment of other PIC components. However, Gal4VP16 was also able to recruit nearly wild-type levels of Rpb3p, Srb4p, and TFIIB in the absence of rTBP, as noted previously (Fig. 2, lane 14; Ranish et al. 1999). This is because Gal4-VP16, unlike Gal4-AH, can recruit holo- 


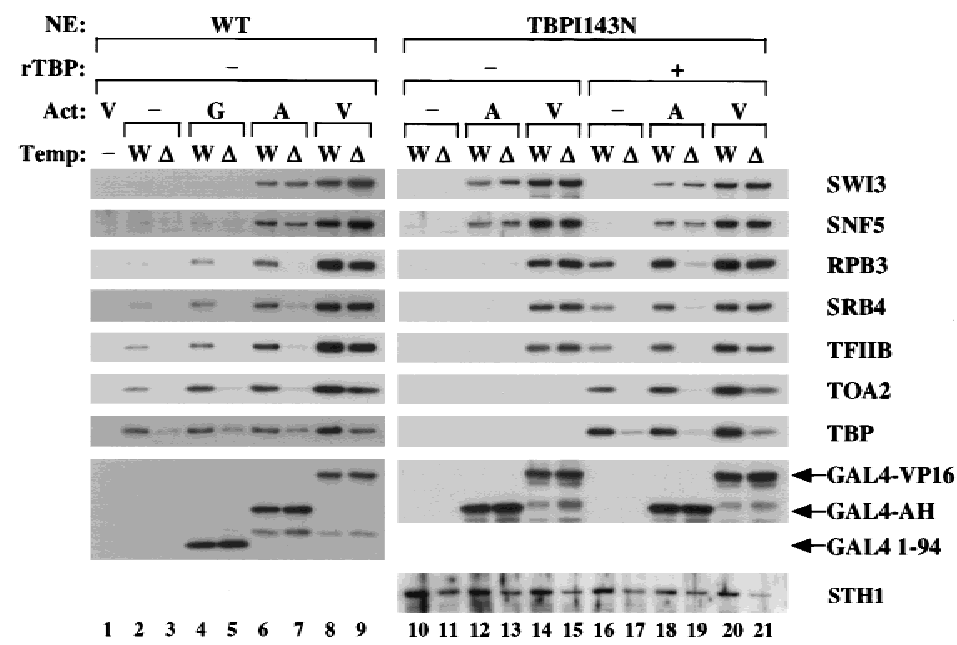

Figure 2. SWI/SNF is recruited to DNA by activators independently of promoter sequences. PICs were assembled on both wild-type $(\mathrm{W})$ and $\Delta$ Promoter $(\Delta)$ templates as stated in Fig. 1, with the nuclear extracts (NE) indicated. Reactions were performed either with no activator $(-)$, the GAL4 (1-94) DNA-binding domain (G), or Gal4-AH (A) and Gal4-VP16 $(\mathrm{V})$, as indicated. rTBP (400 ng) was added where indicated. PICs were analyzed by Western blotting with antibodies against the components indicated at right. (Lane 1) Dynabeads without templates were used in PIC assembly as a control for nonspecific binding to beads.

enzyme in the absence of TBP. By using GST-Swi3p to create a standard curve for Western blots, we determined that $\sim 5$ fmoles of SWI/SNF were recruited to the promoter by Gal4-AH (data not shown). This is significantly lower than the $\sim 40$ fmoles of TFIIA, TFIIB, and TFIIE and $\sim 200$ fmoles of TBP recruited by Gal4-AH in this assay (Ranish 1999). Thus, it is possible that SWI/ SNF is not recruited to promoters as part of the PIC, because only $\sim 10 \%$ of the templates are occupied by PICs in this assay (Ranish 1999). Together, these data are consistent with the idea that SWI/SNF is recruited to promoters independent of holoenzyme.

Next, we determined whether SWI/SNF recruitment by activators required the presence of promoter sequences. The HIS4 promoter sequences from the wildtype template were deleted and replaced by nonpromoter DNA to form the $\Delta$ Promoter template (Fig. 1A). We then tested this template in immobilized template assays using a wild-type NE. Swi3p and Snf5p recruitment by Gal4-AH and Gal4-VP16 was completely unaffected by deletion of the promoter sequences (Fig. 2, lanes 3,7,9). As expected, only small-to-undetectable amounts of the other general factors probed for were present when Gal4$\mathrm{AH}$ was used with this template, whereas nearly wildtype amounts of holoenzyme components were recruited by Gal4-VP16. Identical results were seen when the TBPI143N mutant extract was used (Fig. 2, lanes $11,13,15)$. As before, the recruitment of Swi3p and Snf5p was unaffected by the addition of rTBP (Fig. 2, lanes $17,19,21)$.

We also performed an immobilized template assay using a template that terminated 14-bp downstream of the Gal4 site. Digestion of this template with PstI after in- cubation with NE produced a 53-bp fragment. Although approximately twofold less Gal4-AH bound to this template, we saw no recruitment of SWI/SNF (data not shown). These data, together with evidence that SWI/SNF can only bind nonspecifically to pieces of DNA >80 bp (Quinn et al. 1996), suggest that the formation of a stable complex between SWI/SNF and activator requires DNA, but not specific promoter sequences.

\section{Transcriptional activators can target SWI/SNF remodeling activity}

Next, we wished to determine whether a transcriptional activator could target the chromatin remodeling activity of purified SWI/SNF complex. To investigate this possibility, we exploited a sensitive nucleosomal array remodeling assay in which the activity of a nucleosome remodeling complex is coupled to restriction enzyme activity (Logie and Peterson 1997, 1999). The DNA template that we used is composed of 11 head-to-tail repeats of a $L$. variegatus 5S rRNA gene (208-11S; Fig. 3A). Each repeat can position a nucleosome after in vitro reconstitution with purified histone octamers, yielding a homogeneous array of positioned nucleosomes. The sixth repeat of this DNA template contains a $5 \mathrm{~S}$ sequence that bears a unique SalI-HincII restriction site close to the dyad axis of symmetry of the reconstituted nucleosome (Polach and Widom 1995), thereby allowing for a quantitative kinetic assay for nucleosome remodeling within nucleosomal arrays (Logie and Peterson 1999). To investigate whether a GAL4 activator can target SWI/SNF remodeling activity, we modified the DNA template so that it contains five high affinity GAL4-binding sites adjacent to the $5 \mathrm{~S}$ repeat that harbors the SalI-HincII restriction site (208-11S-GAL4; Fig. 3A). Reconstitution of the 208-11S-GAL4 DNA template into a nucleosomal array positions the GAL4binding sites in the linker region between two positioned nucleosomes (Fig. 3A).

We assembled four sets of remodeling reactions in which targeting of remodeling activity would occur on radiolabeled arrays competing against a 15 -fold excess of unlabeled competitor arrays. (Fig. 3B). HincII digestion of these four array mixtures was essentially equivalent in the absence of SWI/SNF [(Fig. 3B) first order rate $=1 \times 10^{-3} / \mathrm{min}$; similar results are seen in the presence of SWI/SNF but in the absence of ATP (Logie and Peterson 1997; Logie et al. 1999)]. Then, we assessed the capacity of SWI/SNF to remodel the labeled nucleosomal arrays in the absence of a GAL4 derivative. Addition of a limiting concentration of SWI/SNF complex (0.8 nM) stimulated HincII kinetics three- to fivefold (first order rate $=3 \times 10^{3}$ to $5 \times 10^{-3} / \mathrm{min}$ ). Thus, SWI/ SNF can remodel both types of nucleosomal arrays, and the presence or absence of GAL4-binding sites on the labeled or competitor array does not influence the kinetics (Fig. 3B; data not shown). Next, we tested whether Gal4-VP16 could target SWI/SNF activity to an array 
A

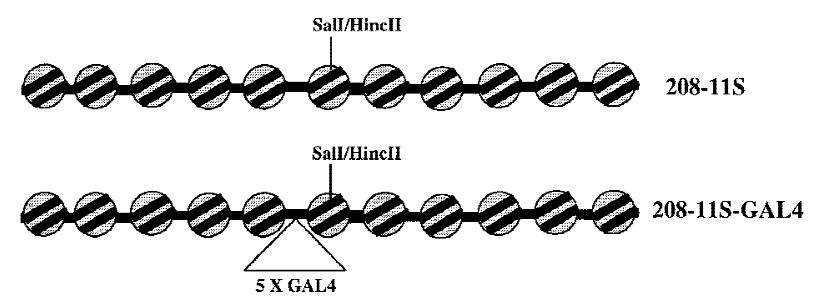

B

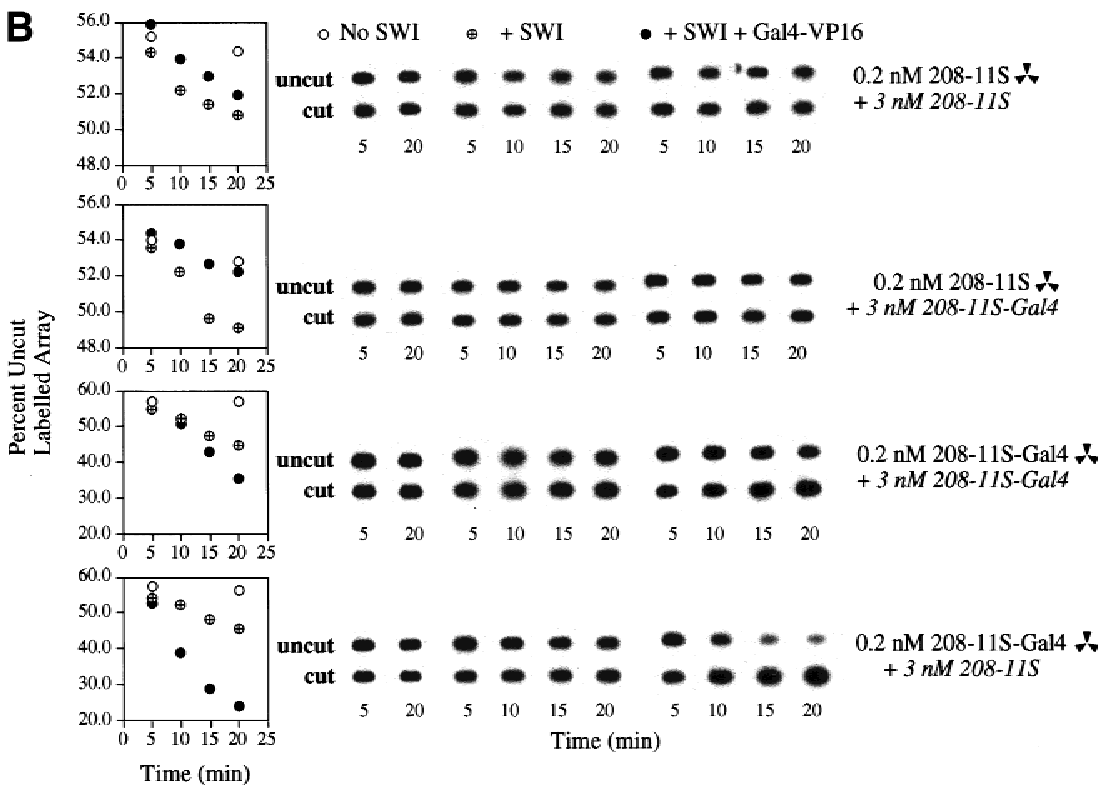

Figure 3. SWI/SNF remodeling activity can be targeted by a transcriptional activator. (A) Schematic of nucleosomal array templates. $(B)$ The ${ }^{32}$ P-labeled arrays $(0.2 \mathrm{nM}$ final) were mixed with unlabeled arrays ( $3 \mathrm{~nm}$ final) in the four possible combinations and then either incubated with HincII alone (O) or exposed to $0.8 \mathrm{nM} \mathrm{SWI/SNF}$ and HincII in the absence (crossed circles), or presence of $10 \mathrm{~nm}$ GAL4-VP16 (-). The digestion of the labeled array with time was quantified and graphed at left. $\left(23 \times 10^{-3} / \mathrm{min}\right.$; Fig. $3 \mathrm{~B}$, bottom $)$. This stimulation of restriction enzyme cleavage rate by activators was absolutely dependent on the continuous presence of ATP (data not shown; see also Logie and Peterson 1997) and on the presence of SWI/SNF complex (Fig. 4A). Together, these experiments demonstrate that the Gal4-VP16 activator can recruit SWI/ SNF remodeling activity in vitro to nucleosomal arrays bearing GAL4-binding sites.

\section{Recruitment of SWI/SNF activity requires an acidic activation domain}

Next we tested the effects of four different GAL4 derivatives on remodeling kinetics-GAL4 (1-94), GAL4-AH, GAL4VP16, and the nonacidic activator, GAL4-proline. Each reaction contained 0.2 nm labeled 208-11S-GAL4 array and 3 nM unlabeled 208-11S competitor array. Under these conditions, SWI/SNF remodeling is barely detectable (HincII cleavage rate $\left.1-2 \times 10^{-3} / \mathrm{min}\right)$. Addition of $10 \mathrm{~nm}$ GAL4-AH or GAL4-VP16 increased the apparent first order rate of SWI/SNF remodeling in this experiment more than sixfold $\left(14-17 \times 10^{-3} / \mathrm{min}\right)$. However, the presence of GAL4(1-94) or GAL4-proline-even when present at $60 \mathrm{~nm}$ (data not shown)- had no effect on the first order rate of cleavage $\left(1-2 \times 10^{-3} /\right.$ min; Fig. 4A). Thus, targeting of SWI/ SNF nucleosome remodeling activity requires an acidic activation domain.

We have shown that SWI/SNF can be bearing Gal4 sites. This was achieved by including $10 \mathrm{~nm}$ Gal4-VP16 in the four array mixtures. The presence of Gal4-VP16 did not affect remodeling kinetics when both the labeled and unlabeled arrays lacked Gal4 sites (first order cleavage rate $=3 \times 10^{-3} / \mathrm{min}$; Fig. $3 \mathrm{~B}$, top). When remodeling of the labeled array lacking Gal4 sites (20811S) was measured in the presence of a 15-fold excess of unlabeled array bearing Gal4 sites (208-11S-GAL4), we observed a $\sim 3$-fold reduction in the first order rate of restriction enzyme cleavage induced by Gal4-VP16 $\left(1 \times 10^{-3} / \mathrm{min}\right.$; Fig. $3 \mathrm{~B}$, second panel $)$, indicating that the Gal4-VP16 that was bound to the competitor array sequestered SWI/SNF activity. When both the labeled and competitor array bore GAL4 sites, we observed an approximately two- to threefold increase in rate compared with the rate in the absence of Gal4-VP16 $\left(5 \times 10^{-3}\right.$ vs. $14 \times 10^{-3} / \mathrm{min}$; Fig. 3B, third panel). Finally, when $10 \mathrm{~nm}$ Gal4-VP16 was added to reactions containing a labeled array bearing GAL4 sites (208-11S-GAL4) immersed in a 15 -fold excess of unlabeled array lacking GAL4 sites (208-11S), we observed a dramatic enhancement of the cleavage rate when compared with SWI/SNF alone recruited from a NE by a DNA-bound activator in the absence of an obligatory association with a Pol II holoenzyme or TBP. Furthermore, an activator can target SWI/SNF activity in reactions that contain only DNA, histones, SWI/SNF, and the activator protein. These results are consistent with the hypothesis that SWI/SNF is recruited in vivo by direct interactions with gene-specific activators. SWI/SNF may only be recruited by activators that control expression of the small number of SWI/SNF-dependent genes, or, alternatively, SWI/SNF may be recruited to many genes even though SWI/SNF activity might not be rate determining for expression. Our data does not rule out the possibility that SWI/SNF can be recruited to target genes via other mechanisms. In fact, recently it has been shown that recruitment of SWI/ SNF to the HTA1-HTB1 locus requires HIR1 and HIR2, which encode transcriptional repressors (Dimova et al. 1999).

We also found that the activator-dependent recruitment of SWI/SNF to an immobilized template required $>53 \mathrm{bp}$ of DNA adjacent to the GAL4-binding site. These results indicate that SWI/SNF may not interact stably 

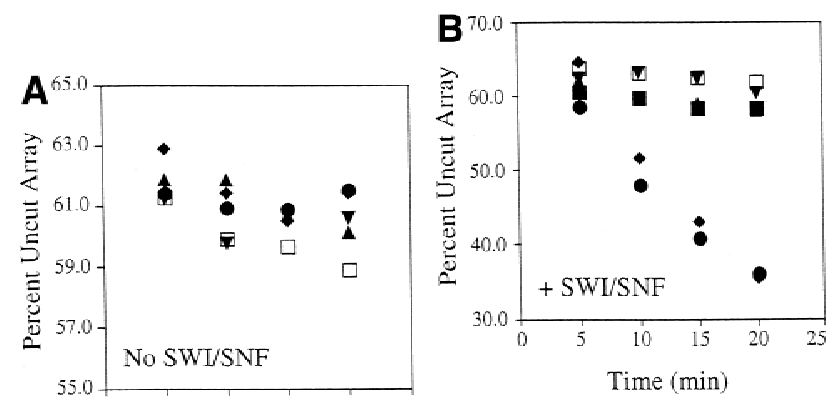

Figure 4. SWI/SNF activity is recruited by acidic activation domains. HincII cleavage kinetics of reactions containing 0.2 nм labeled 208-11S-GAL4 array, 3 nм unlabeled 208-11S competitor array in the presence or absence of GAL4 derivatives, and in the absence $(A)$ or presence $(B)$ of $0.8 \mathrm{nM} \mathrm{SWI}$ /SNF. Note that the GAL4 derivatives did not affect HincII cleavage kinetics in the absence of SWI/SNF. ( $\square$ ) No Gal4/no SWI/SNF; (

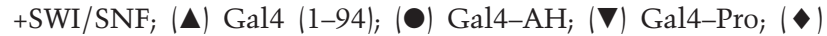
Gal4-VP16.

with activators in solution, but that SWI/SNF is targeted by a DNA-bound activator. This possibility is consistent with the observation that recruitment of SWI/SNF to the HO locus requires SWI5, which binds without the assistance of SWI/SNF to two sites located between positioned nucleosomes (Cosma et al. 1999; J. Krebs and C.L. Peterson, unpubl.). SWI/SNF might also be targeted by transcription factors bound to nucleosomes because some transcription factors are able to occupy nucleosomal sites (Workman and Kingston 1992; Li and Wrange 1993). Alternatively, other remodeling activities may facilitate the binding of an activator to a nucleosomal site, which would then allow subsequent recruitment of SWI/ SNF.

How do activators coordinate interactions with the myriad of transcription factors that can serve as regulatory targets? In the case of chromatin remodeling factors, an acidic activation domain can target either the ATPdependent SWI/SNF complex (this work) or the SAGA histone acetyltransferase (Utley et al. 1998). The ability of activators to recruit either SWI/SNF or SAGA may explain the functional redundancy of these two complexes in vivo (Pollard and Peterson 1997; Roberts and Winston 1997; Biggar and Crabtree 1999). However, in the case of the $H O$ gene, whose expression is absolutely dependent on both SWI/SNF and SAGA (Pollard and Peterson 1997), it appears that the SWI5 activator can recruit SWI/SNF, but not SAGA complex. Thus, whereas generic activators like GAL4-AH or GAL4-VP16 may be able to interact with all possible targets, different genespecific activators may have a restricted spectrum of binding partners to better coordinate the sequence of events that lead to gene activation.

\section{Materials and methods}

Immobilized templates and PIC assay

Wild-type template was prepared and biotinylated by PCR from pSH515 as described previously (Ranish et al. 1999). The $\Delta$ Promoter template was made by digesting pSH515 with $\mathrm{XhoI}$ and $\mathrm{BamHI}$ to remove a $\sim 150$-bp
HIS4 promoter fragment. The cut plasmid was then purified, filled in with Klenow fragment (GIBCO-BRL), and religated to create pSH515 $\Delta$. The $\Delta$ Promoter template was prepared and biotinylated by PCR from pSH515 $\Delta$ P with the same primers and reaction conditions as for pSH515. The templates were immobilized on M-280 Streptavidin Dynabeads (Dynal) as described previously (Ranish et al. 1999).

The PIC assay was performed in 100- $\mu 1$ reactions as described in Ranish et al. (1999), except that no BSA was included in the transcription buffer used to wash the templates. The wild-type and mutant yeast strains used are described in Ranish et al. (1999). Each reaction contained $480 \mu \mathrm{g}$ of wild type, $480 \mu \mathrm{g}$ of $\Delta \mathrm{Srb} 2$, or $360 \mu \mathrm{g}$ of TBPI143N nuclear extract, as indicated.

Reconstitution of nucleosomal arrays

DNA templates were prepared by restriction enzyme digestion of pCL7c (208-11S) or pCL8b (208-11S-Gal4), purified by gel filtration, and labeled by Klenow fill-in reaction with $\left[\alpha-{ }^{32} \mathrm{P}\right] \mathrm{dATP}$ (Logie and Peterson 1999). Reconstitution of histone octamers onto 208-11 array templates were performed by salt gradient dialysis with a ratio of histone octamer to $5 \mathrm{~S}$ rRNA repeat of 1.0 (Logie and Peterson 1999). Coupled remodeling-restriction reactions were performed in $25 \mu \mathrm{l}$, with a final concentration of $5 \mathrm{~mm} \mathrm{MgCl}_{2}, 125 \mathrm{~mm} \mathrm{NaCl}, 10 \mathrm{~mm}$ Tris-HCL (pH 8.0), 1 mM DTT, 0.1 $\mathrm{mg} / \mathrm{ml} \mathrm{BSA}$, and $1 \mathrm{~mm}$ ATP. HincII (New England Biolabs) was added to reactions at a final concentration of 500 units $/ \mathrm{ml}$. HincII cleavage was quantified by PhosphorImager analysis, and first-order rates were determined by curve fitting. In multiple independent experiments, the firstorder rates of restriction enzyme cleavage for each particular combination of array, remodeler, and activator varied by $\leq 30 \%$.

Protein purification

SWI/SNF complex was purified from yeast strain CY396 (Logie and Peterson 1999) and lacked detectable levels of Pol II holoenzyme as assayed by Western blot analysis with antibodies to RPB1, SRB2, SRB4, and SRB5 (data not shown). In contrast, high levels of these components were detectable early in the fractionation scheme (i.e., the Ni-NTA eluate. GAL4(1-94), GAL4(1-147)-AH, and GAL4(1-147)-VP16 were purified from bacteria as described previously (Lin et al. 1988; Chasman et al. 1989). The GAL4-proline was purified from bacteria with the protocol for GAL4(1-94). The binding activities of GAL4 derivatives were determined by gel shift assays with a 154-bp DNA fragment containing a single, high affinity GAL4-binding site (Côté et al. 1994) in the presence of $3 \mathrm{~nm}$ unlabeled 208-11S-Gal4 array DNA template. In these assays $10 \mathrm{~nm}$ GAL4-AH was required for half-maximal binding. The concentration of each GAL4 derivative was adjusted to yield an equivalent level of DNAbinding activity.

\section{Acknowledgments}

We thank members of the Hahn and Reeder laboratories for help and suggestions, and J. Ranish and T. Tsukiyama for thoughtful discussions. We also thank H. Sakurai for Gall1 antibodies, Nancy Thompson for Rpb3 antibodies, J. Movius for Srb4 antibodies, B. Laurent for Sth1 antibodies, and R. Young for Srb5 antibodies. We also thank J. Ranish for the TBP temperature-sensitive extract, J. Geiger for rTBP, and J. Flanagan for GST-SWI3 protein. We thank T. Imbalzano for the kind gift of GAL4(194) protein and N. Tanese for the GAL4-proline expression plasmid. This work was supported by grants from the National Institutes of Health to C.L.P. (GM496054) and to S.H. (GM42551), a grant from the National Cancer Institute to N.Y. (T32CA09657), and by a fellowship to C.L. from the Human Frontiers Science Organization. C.L.P. is a Scholar of the Leukemia Society of America, and S.H. is an Associate Investigator of the Howard Hughes Medical Institute.

The publication costs of this article were defrayed in part by payment of page charges. This article must therefore be hereby marked 'advertisement' in accordance with 18 USC section 1734 solely to indicate this fact.

\section{References}

Biggar, S.R. and G.R. Crabtree. 1999. Continuous and widespread roles for the Swi-Snf complex in transcription. EMBO J. 18: 2254-2264.

Burns, L. and C.L. Peterson. 1997. Protein complexes for remodeling chromatin. Biochem. Biophys. Acta 1350: 159-168. 
Cairns, B.R., Y. Lorch, Y. Li,, M. Zhang, L. Lacomis, H. Erdjument-Bromage, P. Tempst, J. Du, B. Laurent, and R.D. Kornberg. 1996. RSC, an essential, abundant chromatin-remodeling complex. Cell 87: 12491260 .

Chasman, D.I., J. Leatherwood, M. Carey, M. Ptashne, and R.D. Kornberg. 1989. Activation of yeast polymerase II transcription by Herpesvirus VP16 and GAL4 derivatives in vitro. Mol. Cell. Biol. 9: 4746-4749.

Cho, H., G. Orphanides, X. Sun, X.J. Yang, V. Ogryzko, E. Lees, Y. Nakatani, and D. Reinberg. 1998. A human RNA polymerase II complex containing factors that modify chromatin structure. Mol. Cell. Biol. 18: $5355-5363$

Cosma, M.P., T. Tanaka, and K. Nasmyth. 1999. Ordered recruitment of transcription and chromatin remodeling factors to a cell cycle-and developmentally regulated promoter. Cell 97: 299-311.

Côté, J., J. Quinn, J. Workman, and C.L. Peterson. 1994. Stimulation of GAL4 derivative binding to nucleosomal DNA by the yeast SWI/SNF protein complex. Science 265: 53-60.

Dimova, D., N. Zeena, S. Furgeson, S. Eguchi, and M.A. Osley. 1999. A role for transcriptional repressors in targeting the yeast Swi/Snf complex. Mol. Cell 4: 75-83.

Georgakopoulos, T. and G. Thireos. 1992. Two distinct yeast transcriptional activators require the function of the GCN5 protein to promote normal levels of transcription. EMBO J. 11: 4145-4152.

Grant, P.A., L. Duggan, J. Cote, S.M. Roberts, J.E. Brownell, R. Candau, R. Ohba, T. Owen-Hughes, C.D. Allis, F. Winston, S.L. Berger, and J.L. Workman. 1997. Yeast Gen5 functions in two multisubunit complexes to acetylate nucleosomal histones: Characterization of an Ada complex and the SAGA (Spt/Ada) complex. Genes \& Dev. 11: 16401650.

Holstege, F.C., E.G. Jennings, J.J. Wyrick, T.I. Lee, C.J. Hengartner, M.R Green, T.R. Golub, E.S. Lander, and R.A. Young. 1998. Dissecting the regulatory circuitry of a eukaryotic genome. Cell 95: 717-728.

Kruger, W., C.L. Peterson, A. Sil, C. Coburn, G. Arents, E.N. Moundrianakis, and I. Herskowitz. 1995. Amino acid substitutions in the structured domains of histones $\mathrm{H} 3$ and $\mathrm{H} 4$ partially relieve the requirement of the yeast SWI/SNF complex for transcription. Genes \& Dev. 9: 2770-2779.

Li, Q. and O. Wrange. 1993. Translational positioning of a nucleosomal glucocorticoid response element modulates glucocorticoid receptor affinity. Genes \& Dev. 7: 2471-2482.

Lin, Y.-S., M.F. Carey, M. Ptashne, and M.R. Green. 1988. GAL4 derivatives function alone and synergistically with mammalian activators in vitro. Cell 54: 659-664.

Logie, C. and C.L. Peterson. 1997. Catalytic activity of the yeast SWI/ SNF complex on nucleosome arrays. EMBO J. 16: 6772-6782.

-1999. Purification and biochemical properties of yeast SWI/SNF complex. Methods in Enzymol. 304: 726-741.

Logie, C., C. Tse, J.C. Hansen, and C.L. Peterson. 1999. The core histone $\mathrm{N}$-terminal domains are required for multiple rounds of catalytic chromatin remodeling by the SWI/SNF and RSC complexes. Biochemistry 38: 2514-2522.

Marcus, G.A., N. Silverman, S.J. Berger, J. Horiuchi, and L. Guarente. 1994. Functional similarity and physical association between GCN5 and ADA2: Putative transcriptional adaptors. EMBO $J$. 13: $4807-4815$

Myers, L.C., C.M. Gustafsson, D.A. Bushnell, M. Lui, H. ErdjumentBromage, P. Tempst, and R.D. Kornberg. 1998. The Med proteins of yeast and their function through the RNA polymerase II carboxyterminal domain. Genes \& Dev. 12: 45-54.

Neish, A.S., S.F. Anderson, B.P. Schlegel, W. Wei, and J.D. Parvin. 1998 Factors associated with the mammalian RNA polymerase II holoenzyme. Nucleic Acids Res. 26: 847-853.

Owen-Hughes, T.A., R.T. Utley, J. Cote, C.L. Peterson, and J.L. Workman. 1996. Persistent site-specific remodeling of a nucleosome array by transient action of the SWI/SNF complex. Science 273: 513-516.

Polach, K.J. and J. Widom. 1995. Mechanism of protein access to specific DNA sequences in chromatin: A dynamic equilibrium model for gene regulation. J. Mol. Biol. 254: 130-149.

Pollard, K.J. and C.L. Peterson. 1997. Role for ADA/GCN5 products in antagonizing chromatin-mediated transcriptional repression. Mol. Cell. Biol. 17: 6212-6222.

Quinn, J., A. Fyrberg, R.W. Ganster, M.C. Schmidt, and C.L. Peterson.
1996. DNA-binding properties of the yeast SWI/SNF complex. $\mathrm{Na}$ ture 379: 844-847.

Ranish, J.A., N. Yudkovsky, and S. Hahn. 1999. Intermediates in formation and activity of the RNA polymerase II preinitiation complex: Holoenzyme recruitment and a postrecruitment role for the TATA box and TFIIB. Genes \& Dev. 13: 49-63.

Reddy P. and S. Hahn. 1991. Dominant negative mutations in yeast TFIID define a bipartite DNA-binding region. Cell 65: 349-357.

Roberts, S.M. and F. Winston. 1997. Essential functional interactions of SAGA, a Saccharomyces cerevisiae complex of Spt, Ada, and Gcn5 proteins, with the Swi/Snf and Srb/Mediator complexes. Genetics 147: 451-465

Saleh, A., V. Lang, R. Cook, and C.J. Brandl. 1997. Identification of native complexes containing the yeast coactivator/repressor proteins NGG1/ADA3 and ADA2. J. Biol. Chem. 272: 5571-5578.

Tsukiyama, T., J. Palmer, C.C. Landel, J. Shiloach, and C. Wu. 1999. Characterization of the imitation switch subfamily of ATP-dependent chromatin-remodeling factors in Saccharomyces cerevisiae. Genes \& Dev. 13: 686-697.

Utley, R.T., J. Cote, T. Owen-Hughes, and J.L. Workman. 1997. SWI/SNF stimulates the formation of disparate activator-nucleosome complexes but is partially redundant with cooperative binding. J. Biol. Chem. 272: 12642-12649.

Utley, R.T., K. Ikeda, P.A. Grant, J. Cote, D.J. Steger, A. Eberharter, S John, and J.L. Workman. 1998. Transcriptional activators direct histone acetyltransferase complexes to nucleosomes. Nature 394: 498502.

Wilson, C.J., D.M. Chao, A.N. Imbalzano, G.R. Schnitzler, R.E. Kingston, and R.A. Young. 1996. RNA polymerase II holoenzyme contains SWI/SNF regulators involved in chromatin remodeling. Cell 84: 235 244.

Winston, F. and M. Carlson. 1992. Yeast SNF/SWI transcriptional activators and the SPT/SIN chromatin connection. Trends Genet 8: $387-391$.

Workman, J.L. and R.E. Kingston. 1992. Nucleosome core displacement in vitro via a metastable transcription factor-nucleosome complex. Science 258: 1780-1784.

Yoshinaga, S.K., C.L. Peterson, I. Herskowitz, and K. Yamamoto. 1992 Roles of SWI1, SWI2, and SWI3 proteins for transcriptional enhancement by steroid receptors. Science 258: 1598-1604. 


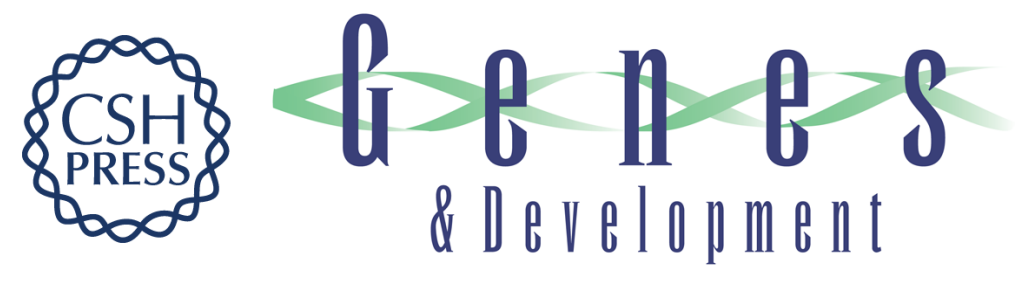

\section{Recruitment of the SWI/SNF chromatin remodeling complex by transcriptional activators}

Natalya Yudkovsky, Colin Logie, Steven Hahn, et al.

Genes Dev. 1999, 13:

References This article cites 35 articles, 18 of which can be accessed free at:

http://genesdev.cshlp.org/content/13/18/2369.full.html\#ref-list-1

License

Email Alerting Receive free email alerts when new articles cite this article - sign up in the box at the top Service right corner of the article or click here.

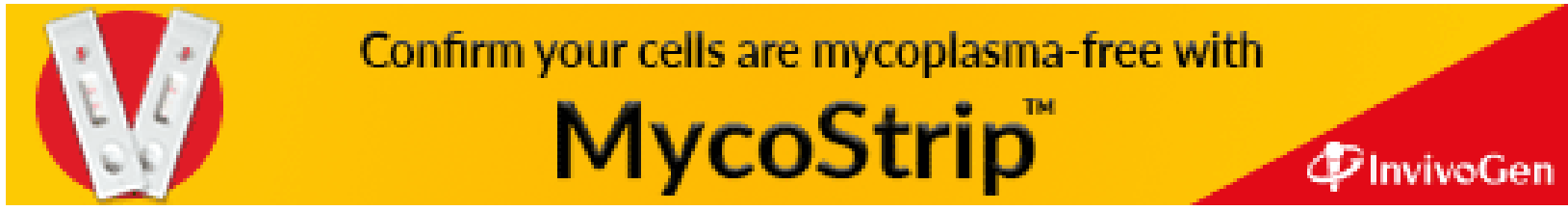

